\title{
Runde Formen über semilokalen Ringen *
}

\author{
MANFRED KNEBUSCH
}

Herrn Ernst Witt zu seinem 60. Geburtstag am 26. Juni 1971 gewidmet

Das Analogon zu der von A. Pfister entdeckten Tatsache, daß eine quadratische Form $\left(1, a_{1}\right) \otimes \cdots \otimes\left(1, a_{k}\right)$ über einem Körper für beliebiges $k$,rund“ ist ([10], Theorem 1), bleibt über jedem lokalen Ring $C$ sowohl für nichtausgeartete quadratische als auch für nichtausgeartete symmetrisch bilineare Formen richtig. Wir werden dies sogar über nahezu jedem semilokalen Ring beweisen, s. Theorem 1.5. Falls $C$ lokal mit 2 Einheit ist, werden wir daraus folgern können, da $\beta$ die Stufe von $C$, d. h. die kleinste Anzahl von Quadraten in $C$, die man benötigt, um -1 als Quadratsumme darzustellen, eine 2-Potenz ist, sofern -1 überhaupt Quadratsumme ist (vgl. [9, 10]). Ist 2 nicht Einheit, so braucht dies nicht wahr zu sein, wie schon $C=\mathbb{Z} / 4 \mathbb{Z}$ zeigt. Doch können im lokalen Falle für die Stufe neben $2^{r}, \infty$ höchstens noch Werte $2^{r}-1$ auftreten.

Weiter verallgemeinern wir den Satz von Witt [unveröffentlicht], daß das Annullatorideal eines runden quadratischen Raumes binär erzeugt wird, auf semilokale Ringe. Leider scheint über die Annullatorideale der runden bilinearen Räume über lokalen Ringen, in denen 2 nicht Einheit ist, nichts bekannt zu sein.

Aus der Tatsache, da $\beta$ die Formen $\left(1, a_{1}\right) \otimes \cdots \otimes\left(1, a_{k}\right)$ rund sind, lassen sich auch über semilokalen Ringen die bekannten Folgerungen über die Struktur des Wittringes $W(C)$ ziehen $([11]$, vgl. $[7,14])$. Wir werden uns hierüber jedoch kurz fassen, weil es inzwischen einen elementareren Zugang zu Pfisters Struktursätzen über semilokalen Ringen gibt, s. [3].

Alle in dieser Arbeit wiedergegebenen Beweise wurden 1967/68 über Körpern von Witt gefunden. Das meiste wurde inzwischen in [7] und [14] dargestellt. Bei der Übertragung der Wittschen Beweise auf semilokale oder auch nur lokale Ringe treten technische Komplikationen auf. Der Leser möge sich klar machen, wie elegant und überaus kurz alle Beweise werden, wenn man sich auf den Körperfall beschränkt.

Ich danke Herrn Prof. Witt herzlich für anregende Gespräche und Einsicht in seine Manuskripte.

Bezeichnungen. $C$ ist stets ein semilokaler Ring, d.h. ein kommutativer Ring mit Einselement, der nur endlich viele maximale Ideale $m_{1}, \ldots m_{t}$ besitzt. Wir setzen ohne wesentliche Einschränkung der Allgemeinheit immer voraus,

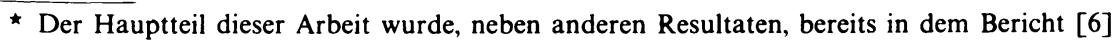
skizziert. 
$\mathrm{da} B C$ keine Idempotenten $\neq 0,1$ besitzt. Mit $\mathfrak{r}$ bezeichnen wir das Radikal (= Durchschnitt der $\mathrm{m}_{i}$ ), mit $C^{*}$ die Einheitengruppe von $C$. Unter einem (symmetrisch) bilinearen Raum $E$ über $C$ verstehen wir einen freien, endlich erzeugten $C$-Modul $E$, versehen mit einer symmetrischen bilinearen Form $B: E \times E \rightarrow C$. Wir beschreiben $E$ häufig durch die Wertematrix $\left(B\left(e_{i}, e_{j}\right)\right) \mathrm{zu}$ einer Basis $e_{1}, \ldots e_{n}$. Der Raum $E$ (oder die Form $B$ ) heiße nicht entartet, falls die Determinante dieser Matrix eine Einheit ist. Ein binärer nichtentarteter Raum hat eine Matrix $\left(\begin{array}{ll}\alpha & 1 \\ 1 & \beta\end{array}\right)$, für die wir kurz $A(\alpha, \beta)$ schreiben. Dabei muß $1-\alpha \beta \in C^{*}$ sein. Ein bilinearer Raum $E$ heiße eigentlich, falls $E$ nichtentartet ist und das von den Werten $B(x, x)$ mit $x \in E$ in $C$ erzeugte Ideal mit $C$ übereinstimmt. Dann besitzt $E$ eine Orthogonalbasis, d.h. ist zu einem Raum $\left(\lambda_{1}\right) \perp \cdots \perp\left(\lambda_{n}\right)$ mit $\lambda_{i} \in C^{*}$ isomorph ([4], Lemma 5.4.1 $\left.{ }^{1}\right)$. Für eine solche orthogonale Summe schreiben wir auch $\left(\lambda_{1}, \ldots, \lambda_{n}\right)$. Der Raum $E$ heißt metabolisch, falls $E$ orthogonale Summe binärer Räume der Gestalt $A(a, 0)$ ist (vgl. [4]), hyperbolisch, falls dabei überdies alle Koeffizienten $a=0$ gewählt werden können. \{Ist $2 \in C^{*}$, so ist jeder metabolische Raum hyperbolisch, s. [4].\}

Unter einem quadratischen Raum $E$ über $C$ verstehen wir einen freien endlich erzeugten $C$-Modul $E$, versehen mit einer quadratischen Form $q$, d. h. einer Abbildung $q: E \rightarrow C$, so daß für $x, y \in E, \lambda \in C$ gilt: $q(\lambda x)=\lambda^{2} q(x), q(x+y)$ $=q(x)+q(y)+B(x, y)$ mit bilinearer Form $B$. Wir beschreiben $E$ oft durch die Wertetabelle $\left[a_{i j}\right], a_{i i}:=q\left(e_{i}\right), a_{i j}:=B\left(e_{i}, e_{j}\right)$ für $i \neq j$, zu einer Basis $\left\{e_{i}\right\}$ von $E$ über $C$. Der quadratische Raum $E$ heiße nicht entartet, falls $B$ nicht entartet ist. Ein nicht entarteter binärer quadratischer Raum hat eine Wertetabelle $\left[\begin{array}{ll}\alpha & 1 \\ 1 & \beta\end{array}\right]$. Dafür schreiben wir kurz $A[\alpha, \beta]$. Dabei muß $1-4 \alpha \beta \in C^{*}$ sein. Orthogonale Summe von Kopien von $A[0,0]$ bezeichnen wir als hyperbolische Räume. Neben dem Tensorprodukt bilinearer Räume ([2], § 1, Def. 11) haben wir auch zu einem bilinearen Raum $\left(E, B_{1}\right)$ und einem quadratischen $\operatorname{Raum}\left(F, q_{2}\right)$ ein Tensorprodukt $E \otimes F$. Dies ist der Modul $E \otimes_{c} F$, versehen mit der quadratischen Form $q$, die folgendermaßen charakterisiert werden kann: $q(x \otimes y)$ $=B_{1}(x, x) q_{2}(y)$ für beliebige $x \in E, y \in F$; die Bilinearform zu $q$ ist das Tensorprodukt $B_{1} \otimes B_{2}$ (z. B. [1], S. 145). Ist $2 \in C^{*}$, so stammt jede symmetrische Bilinearform $B$ von genau einer quadratischen Form $q$ her, nämlich $q(x)$ $=1 / 2 B(x, x)$, und es besteht kein Unterschied zwischen bilinearen und quadratischen Räumen.

Für die quadratischen bzw. bilinearen Formen aller auftretenden Räume schreiben wir unterschiedslos $q$ bzw. $B$, solange keine Verwirrung entstehen kann. Die zu einer symmetrischen Bilinearform $B$ gehörige Normform $B(x, x)$ bezeichnen wir im allgemeinen mit $n(x)$. Die orthogonale Summe von $r$ Kopien

1 Wie mir verschiedene Herren freundlicherweise mitteilten, fehlt in der letzten Aussage des Lemmas 5.4.1 und einem Teil von $\S 5.5$ aus [4] irrtümlich die Voraussetzung, daß $C$ keine nichttrivialen Idempotente hat. 
eines Raumes $E$ bezeichnen wir mit $r \times E$. Alle in dieser Arbeit betrachteten Räume werden stillschweigend als nicht entartet vorausgesetzt, sofern nicht ausdrücklich etwas anderes gesagt wird. Ein Raum $E$ heiße isotrop, falls er einen Vektor $x$ mit $q(x)=0$ bzw. $n(x)=0$ enthält, der sich zu einer Basis von $E$ ergänzen läßt. Sonst heiße $E$ anisotrop.

\section{§1. Konstruktion runder Räume}

Sei $E$ quadratischer oder bilinearer Raum. $E^{*}$ bezeichne die Menge der strikt anisotropen Vektoren, d. h. der $x \in E$ mit $q(x) \in C^{*}$ bzw. $n(x) \in C^{*}$. Mit $N(E)$ bezeichnen wir die Gruppe der Ähnlichkeitsnormen von $E$, d. h. die Gruppe aller $\lambda \in C^{*}$ mit $E \cong(\lambda) \otimes E$. Wir nennen mit Witt $E$ rund, falls $q\left(E^{*}\right)=N(E)$ bzw. $n\left(E^{*}\right)=N(E)$ ist.

$N$.B. Allgemein gilt für bilineares $E$ : Ist $1 \in n\left(E^{*}\right)$, so ist $N(E) \subset n\left(E^{*}\right)$. Ist $n\left(E^{*}\right) \subset N(E), n\left(E^{*}\right) \neq \emptyset$, so ist $1 \in n\left(E^{*}\right)$, also $E$ rund. Entsprechend für quadratisches $E$.

Beispiele 1.1. (vgl. [7], S. 23, 24). Ein binärer bilinearer oder quadratischer Raum ist genau dann rund, wenn er die 1 darstellt. Runde Räume $E$ einer ungeraden Dimension $n>1$ können nur selten auftreten: $\mathrm{Da} N(E)=C^{* 2}$ ist (man betrachte die Determinante von $E$ ), $\mathrm{mu} B E \cong n \times(1)$ sein und überdies jede Einheit von $C$, die sich als Summe von $n$ Quadraten schreiben läßt, selbst Quadrat sein. Zumindest bei lokalem $C$ folgt daraus leicht, daß überhaupt jede Einheit, die Quadratsumme ist, ein Quadrat ist. Das spätere Kor. 1.4 zeigt, $\mathrm{da} \beta$ dies auch bei semilokalem $C$ sicher richtig ist, wenn alle $\left|C / \mathrm{m}_{i}\right|>3$ sind.

Satz 1.2. Die Anzahl $\left|C / m_{i}\right|$ der Elemente jedes Restklassenkörpers $C / m_{i}$ möge mindestens 3 sein. Dann gilt für beliebiges $a \in C^{*}:$ Ist $F$ ein runder bilinearer oder quadratischer Raum, so ist $(1, a) \otimes F$ ebenfalls rund.

Für den Beweis benötigen wir

Hilfssatz 1.3. Sei $F$ quadratischer oder bilinearer Raum, $\lambda, \mu \in N(F), a \in C^{*}$, $\xi, \eta \in C$. Ist $\varepsilon:=\xi^{2} \lambda+\eta^{2} a \mu$ Einheit, so ist $\varepsilon$ Ähnlichkeitsnorm von $(1, a) \otimes F$.

Beweis (Witt). Es ist

$$
(\lambda, a \mu) \cong(\varepsilon) \otimes(1, a \lambda \mu) .
$$

Man tensoriere beide Seiten mit $F$.

Wir brauchen später Satz 2.2 vor allem für den Fall, daß alle $C / \mathrm{m}_{i}$ mindestens 7 Elemente enthalten. Das wird im Teil a) des folgenden Beweises ohne besondere Mühe gezeigt. Den komplizierteren Teil b) kann der Leser zunächst überschlagen.

Beweis von Satz 1.2. a) Im bilinearen Falle ist zu zeigen, daß für beliebige $x, y F$ das Element $\varrho(x, y):=n(x)+a n(y)$ in $N(E)$ liegt, sofern es Einheit ist. Nach Hilfssatz 1.3 ist es klar, wenn überdies $n(x)$ und $n(y)$ Einheiten sind. Ist dies nicht der Fall, so können wir zu $\varrho(x, y)$ eine Einheit der Gestalt $\xi^{2}+a \eta^{2}$ mit $\xi, \eta \in C$ multiplizieren und brauchen uns nur davon zu überzeugen, da $\beta$ das Produkt in $N(E)$ liegt, denn auch $\xi^{2}+a \eta^{2}$ ist Ähnlichkeitsnorm. Man 
verifiziert sofort, daß

$$
\varrho(x, y)\left(\xi^{2}+a \eta^{2}\right)=\varrho(\xi x-a \eta y, \xi y+\eta x)
$$

ist. Wir wollen $\xi, \eta$ so wählen, da $\beta$ neben $\xi^{2}+a \eta^{2}$ auch $n(\xi x-a \eta y)$ und $n(\xi y+\eta x)$ Einheiten sind. Dann sind wir aufgrund des Hilfssatzes fertig. Wir haben also für jedes maximale Ideal $\mathrm{m}_{i}$ die Bedingungen

$$
\begin{gathered}
\xi^{2}+a \eta^{2} \neq 0 \quad\left(\mathrm{~m}_{i}\right) \\
\xi^{2} n(x)-a \lambda \xi \eta+a^{2} \eta^{2} n(y) \neq 0 \quad\left(\mathrm{~m}_{i}\right) \\
\xi^{2} n(y)+\lambda \xi \eta+\eta^{2} n(x) \neq 0 \quad\left(\mathrm{~m}_{i}\right)
\end{gathered}
$$

zu erfüllen mit der Abkürzung $\lambda=2 B(x, y)$. Nach dem chinesischen Restsatz können wir die Restklassen von $\xi, \eta \bmod m_{i}$ beliebig vorschreiben, haben also nur gewisse Ungleichungen in Körpern zu erfüllen. Ist $n(x) \neq 0, n(y) \neq 0 \bmod \mathrm{m}_{i}$, so wählen wir $\xi \equiv 1, \eta \equiv 0 \bmod m_{i}$. Sei jetzt $n(x) \equiv 0 \bmod m_{i}$, also sicherlich

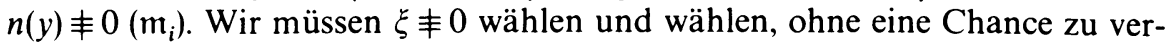
spielen, $\xi \equiv 1$. Dann ist $\bmod m_{i}$ zu erfüllen: $\eta^{2} \neq=a^{-1}, \operatorname{\eta an}(y) \neq z, \eta \neq 0$, $\lambda \eta$ 丰 $-n(y)$. Das geht, falls $\left|C / \mathrm{m}_{i}\right|>5$ ist, und übrigens auch für $C / \mathrm{m}_{i}=\mathbb{F}_{4}$, weil dann $\lambda \equiv 0\left(\mathfrak{m}_{i}\right)$ ist. Ganz entsprechend erledigt man den quadratischen Fall, falls alle $\left|C / \mathfrak{m}_{i}\right|>5$ sind. Man lese überall $q(x)$ statt $n(x)$ und $\lambda=B(x, y)$. Der Fall $C / \mathrm{m}_{i}=\mathbb{F}_{4}$ bleibt jetzt offen.

b) Wir bleiben zunächst beim quadratischen Fall. Durch obige Umformung der vorgegebenen Einheit $q(x)+a q(y)$ konnten wir erreichen, da $\beta$ an allen $m_{i}$ mit $\left|C / \mathrm{m}_{i}\right|>5$ gilt: $q(x)$ 丰 $0, q(y) \neq 0$. An den anderen $\mathrm{m}_{i}$ können wir erreichen, daß wenigstens $q(x) \neq 0$ ist. $\{\xi \equiv 1, \eta \equiv 0$ falls $q(x) \neq 0 ; \xi \equiv 0, \eta \equiv 1$ sonst. $\}$ Damit wird $q(x)$ zu einer Einheit. Da $F$ rund ist, gibt es ein $z \in F$ mit $q(y)=q(x) q(z)$, also

$$
q(x)+a q(y)=q(x)[1+a q(z)] .
$$

Natürlich ist $q(x)$ auch Ähnlichkeitsnorm von $E$. Es fehlt der Nachweis, daß $1+a q(z) \in N(E)$ ist. Nach Hilfssatz 1.3 dürfen wir dazu $1+a q(z)$ mit einer Einheit $1+a \xi^{2} q(w)$ mit $\xi \in C, w \in F^{*}$ multiplizieren. Im folgenden bezeichne $\sigma$ eine Ähnlichkeitstransformation von $F$ mit der Norm $q(w)$. Nun gilt für jedes solche Paar $\xi, w$ :

$$
[1+a q(z)]\left[1+a \xi^{2} q(w)\right]=q\left(x^{\prime}\right)+a q\left(y^{\prime}\right)
$$

mit

$$
x^{\prime}=q(w)^{-1} \sigma(w)-a \xi \sigma(z), \quad y^{\prime}=z+\xi w .
$$

In der Tat ist

$$
\begin{aligned}
& q\left(x^{\prime}\right)=1-a \xi B(z, w)+a^{2} \xi^{2} q(z) q(w), \\
& q\left(y^{\prime}\right)=q(z)+\xi B(z, w)+\xi^{2} q(w) .
\end{aligned}
$$

Durch geeignete Wahl von $\xi \in C$ und $w \in F$ wollen wir erreichen, da $\beta q\left(x^{\prime}\right)$, $q\left(y^{\prime}\right)$ und natürlich $q(w)$ und $1+a \xi^{2} q(w)$ Einheiten sind. Dann sind wir fertig. Nach dem chinesischen Restsatz können wir $w \bmod m_{i} F$ und $\xi \bmod m_{i}$ beliebig 
vorschreiben. Ist $q(z) \neq 0\left(\mathfrak{m}_{i}\right)$, so schreiben wir $\xi \equiv 0\left(\mathfrak{m}_{i}\right)$ und $w \bmod \mathfrak{m}_{i} F$ als irgend einen anisotropen Vektor von $F / \mathrm{m}_{i} F$ vor. Sei jetzt $\mathrm{m}_{i}$ ein maximales Ideal, für das $q(z) \equiv 0\left(\mathrm{~m}_{i}\right)$ ist, also insbesondere $\left|C / \mathrm{m}_{i}\right| \leqq 5$. Wir können $\operatorname{dim} F>2$ voraussetzen, denn für $\operatorname{dim} F \leqq 2$ läßt sich bekanntlich auf $E$ eine (assoziative) Multiplikation $x \cdot y$ erklären, so daß $q(x \cdot y)=q(x) q(y)$ für $x, y \in E$ gilt, und $E$ ist ersichtlich rund. Ist $z \in \mathrm{m}_{i} F$, so kommen wir mit $\xi \equiv 1$ und einer geeigneten Vorschrift für $q(w) \bmod m_{i}$ zum Ziel, denn der Raum $F / m_{i} F$ stellt jedes Element $\neq 0$ von $C / m_{i}$ dar (z. B. [8], S. 157). Sei jetzt $z \notin m_{i} F$ und $\bar{z}$ das Bild von $z$ in $F / \mathrm{m}_{i} F$. Wir können in $\bar{F}=F / \mathrm{m}_{i} F$ eine Zerlegung $\bar{F}=\bar{H} \perp \bar{G}$ finden mit einer hyperbolischen Ebene $\bar{H}$, die $\bar{z}$ enthält. Der Raum $\bar{G}$ hat nicht die Dimension 1. Das ist klar, falls $C / \mathrm{m}_{i}=\mathbb{F}_{4}$ ist, weil $F$ nicht entartet ist. In den verbleibenden Fällen $\left|C / \mathrm{m}_{i}\right|=3,5$ sieht man es so: $F / \mathbf{r} F=\prod_{j} F / \mathrm{m}_{j} F$ ist runder Raum über $C / \mathbf{r}$.

Also ist $F / \mathrm{m}_{i} F$ runder Raum über $C / \mathrm{m}_{i}$. Die Annahme $\operatorname{dim} F=3$ widerspräche unserem Beispiel 1.1, weil in $\mathbb{F}_{3}, \mathbb{F}_{5}$ nicht jede Quadratsumme Quadrat ist. Daher ist der Raum $\bar{G}$ universell. Man wähle nun $\xi \equiv 1 \bmod \mathfrak{m}_{i}$ und $w \bmod \mathfrak{m}_{i} F$ als Element von $\bar{G}$ mit geeigneter Vorschrift für $q(\bar{w})$.

Der bilineare Fall geht entsprechend. Man lese überall $n$ statt $q$ und $2 B$ statt $B$.

q.e.d.

Bei Durchsicht des soeben geführten Beweises stellt man fest, daß nirgends ausgenutzt wurde, da $\beta$ - etwa im quadratischen Falle $-q(x)+a q(y)$ eine Einheit ist, sondern nur, daß für jedes $m_{i}$ mindestens eines der Elemente $q(x), q(y)$ nicht in $\mathfrak{m}_{i}$ liegt. Das führt uns auf ein in $\S 4$ benötigtes

Korollar 1.4. Sei $F$ ein runder quadratischer Raum und $a \in C^{*}$. Weiter seien Vektoren $x, y \in F$ mit $q(x) C+q(y) C=C$ gegeben.

(i) Ist $\operatorname{dim} F>2$ und $\left|C / \mathrm{m}_{i}\right|>2$ für alle $\mathrm{m}_{i}$, so gibt es ein Produkt $c$ von Einheiten der Gestalt $\xi^{2} q(u)+\eta^{2} q(v)$ mit $u, v \in F^{*}$ und $\xi, \eta \in C$, so da $\beta$

$$
c(q(x)+a q(y))=q\left(x^{\prime}\right)+a q\left(y^{\prime}\right)
$$

mit $x^{\prime} \in F^{*}, y^{\prime} \in F^{*}$ ist.

(ii) Dies bleibt auch für $\operatorname{dim} F \leqq 2$ richtig, falls $\left|C / m_{i}\right|>3$ für alle $m_{i}$ ist.

Die Analoga zu (i), (ii) gelten auch für einen runden Bilinearraum $F$. Man lese überall $n$ statt $q$.

Beweis. (i) wurde im Beweis von Satz 1.2 gezeigt, desgl. (ii), sofern sogar $\left|C / m_{i}\right|>5$ für alle $m_{i}$ ist. Um (ii) unter der jetzigen schwächeren Voraussetzung einzusehen, gehen wir den Teil b) des Beweises von Satz 1.2 noch einmal durch. Wir hatten ein vorgegebenes Element $1+a q(z)$ mit einer Einheit $1+a \xi^{2} q(w)$ zu multiplizieren, so daß für alle $m_{i}$ neben $q(w) \neq 0,1+a \xi^{2} q(w)$ 丰 0 noch

$$
\begin{gathered}
1-a \xi B(z, w)+a^{2} \xi^{2} q(z) q(w) \neq 0 \\
q(z)+\xi B(z, w)+\xi^{2} q(w) \neq 0
\end{gathered}
$$

$\operatorname{modm} m_{i}$ erfüllt werden. Jetzt haben wir uns nur noch im Falle $\operatorname{dim} F \leqq 2$ über die Vorschrift an einer Stelle $m_{i}$ mit $\left|C / m_{i}\right|=4$ oder 5 Gedanken zu machen, 
an der überdies $q(z) \equiv 0\left(m_{i}\right)$ ist. Hat $z$ in $F / m_{i} F$ das Bild $\bar{z}=0$, so kommen wir auf die Bedingungen $q(w) \neq 0, \xi$ 列 $0,1+a \xi^{2} q(w) \neq 0$, die sich ersichtlich erfüllen lassen. Sei jetzt $\bar{z} \neq 0$. Dann läßt sich $\bar{z}$ zu einem hyperbolischen Vektorpaar $\bar{z}_{1}=\bar{z}, \bar{z}_{2}$ ergänzen und dieses Vektorpaar spannt $\bar{F}=F / \mathrm{m}_{i} F$ auf. Mit Urbildern $z_{1}=z, z_{2}$ in $F$ setzen wir an: $w=\lambda_{1} z_{1}+\lambda_{2} z_{2}, \xi \equiv 1$, und haben zu erfüllen: $\lambda_{1} \neq 0, \lambda_{2} \neq 0,1+a \lambda_{1} \lambda_{2} \neq 0,1-a \lambda_{2} \neq 0,1+\lambda_{1} \neq 0$. Das ist möglich, falls $\left|C / \mathrm{m}_{i}\right| \geqq 4$ ist.

Der bilineare Fall, in dem nur noch $\mathrm{m}_{i}$ mit $\left|C / \mathrm{m}_{i}\right|=5 \mathrm{zu}$ betrachten sind, geht entsprechend.

Als bilineare Pfister-Räume bezeichnen wir die Räume (1) und $\left(1, a_{1}\right) \otimes \cdots \otimes\left(1, a_{k}\right)$ mit $a_{i} \in C^{*}, k$ beliebig $\geqq 1$. Als quadratischen Pfister-Raum bezeichnen wir das Produkt $P \otimes \mathfrak{B}$ eines beliebigen bilinearen Pfister-Raumes mit einem binären quadratischen Raum $\mathfrak{B}$, der die 1 darstellt.

Theorem 1.5. a) Jeder quadratische Pfisterraum ist rund.

b) Jeder bilineare Pfisterraum $P$ über einem lokalen Ring ist rund.

c) Jeder bilineare Pfisterraum $P$ über einem semilokalen Ring $C$ mit $\left|C / m_{i}\right|>2$ für alle $\mathrm{m}_{i}$ ist rund.

d) Über einem beliebigen semilokalen Ring ist das doppelte $2 \times P$ jedes bilinearen Pfisterraumes rund.

Beispiel 1.6. Für jede 2-Potenz $2^{m}$ ist die Menge $G_{2^{m}}$ aller Einheiten von $C$, die Summen von $2^{m}$ Quadraten sind, über jedem semilokalen Ring $C$ eine Gruppe.

Beweis von Theorem 1.5. Natürlich ist aufgrund von Satz 1.2 nur noch etwas zu zeigen, falls $\left|C / m_{i}\right|=2$ für mindestens ein $m_{i}$ ist. Wir wollen aber Satz 1.2 nur in dem wesentlich einfacheren Falle, da $B$ alle $\left|C / \mathrm{m}_{i}\right|>5$ sind, ausnutzen.

Das Polynom $f(T)=T^{3}+6 T^{2}-T+1$ ist, wie leicht zu sehen, über allen Körpern $\mathbb{F}_{q}$ mit $q \leqq 5$ irreduzibel. Wir betrachten die ganze kubische Erweiterung $D=C[T] /(f(T))=C[t]$ von $C$, wobei $t$ die Restklasse von $T$ bezeichne. Auch $D$ ist ein semilokaler Ring, denn über jedem maximalen Ideal können höchstens 3 maximale Ideale von $D$ liegen. $D$ hat aber keine Restklassenkörper mit weniger als 7 Elementen. Sei nun $E$ ein bilinearer oder quadratischer Pfisterraum über $C$. Dann ist $D \bigotimes_{C} E$ ein Pfisterraum über $D$, also rund. Für ein vorgegebenes Element $\lambda \in n\left(E^{*}\right)$ bzw. $q\left(E^{*}\right)$ ist also $D \bigotimes_{C} E \cong D \bigotimes_{C}[(\lambda) \otimes E]$. Wir betrachten nun die Verlagerung dieser beiden Räume nach $C$ bezüglich der $C$-Linearform $s: D \rightarrow C$, definiert durch $s(1)=1$, $s(t)=s\left(t^{2}\right)=0$ (vgl. [13]), d. h. wir gehen von den D-wertigen Formen dieser Räume durch Hinterschalten von $s$ zu $C$-wertigen quadratischen bzw. bilinearen Formen über. Dadurch ergibt sich eine Isometrie

$$
s_{*}(D) \underset{C}{\bigotimes_{C}} E \cong s_{*}(D) \underset{C}{\bigotimes_{C}}(\lambda) \underset{C}{\bigotimes_{C}} E .
$$

Hier bezeichnet $s_{*}(D)$ den $C$-Vektorraum $D$ mit der Bilinearform $s(x y)$. Dieser Raum hat bezgl. der Basis $1,-t, t^{2}$ die Gestalt $(1) \perp A(0,6)$. Wir erhalten also, $\mathrm{da} ß \lambda$ Ähnlichkeitsnorm des Raumes $E \perp A(0,6) \otimes E=F$ ist. Betrachten wir 
zunächst den quadratischen Fall! $A(0,6) \otimes E$ ist ein hyperbolischer Raum $\cong r \times A[0,0]$ mit $r=\operatorname{dim} E$ (z. B. [1], S. 141, Lemma 2.1).

Wir erhalten

$$
E \perp r \times A[0,0] \cong(\lambda) \otimes E \perp r \times A[0,0] .
$$

Da für quadratische Räume bei semilokalem $C$ die Kürzungsregel gilt ([5], vgl. [12]), ist $E \cong(\lambda) \otimes E$, was zu zeigen war. Sei nun $E$ bilinear. Die von den Normen aller $x \in E$ additiv erzeugte Gruppe $g E$ umfaßt die Normgruppe $\mathfrak{g}(A(0,6) \otimes E)=2 C$. Daher ist $F \cong E \perp r \times A(0,0) \quad$ ([4], Lemma 6.1.2). Wir erhalten also

$$
E \perp r \times A(0,0) \cong(\lambda) \otimes E \perp r \times A(0,0) .
$$

Aufgrund einer Verallgemeinerung des O'Mearaschen Kürzungssatzes ([4], Satz 6.1.3) erhalten wir $E \cong(\lambda) \otimes E$, falls $C$ lokal ist, und auch bei semilokalem $C$, sofern $E$ ein Element $2 \eta$ mit $\eta \in C^{*}$ darstellt. Dies ist sicher der Fall, wenn $E$ das doppelte $2 \times P$ eines Pfisterraumes ist.

q.e.d.

\section{§ 2. Nullteiler und nilpotente Elemente in $W(C)$}

$$
\text { (vgl. }[3,7,11,14])
$$

In diesem Paragraphen seien alle Räume bilinear (und nicht entartet). Wir nennen zwei Räume $E, F$ über unserem semilokalen Ring $C$ äquivalent und schreiben $E \sim F$, wenn es metabolische Räume $M, N$ gibt mit $E \perp M \cong F \perp N$. Die Menge $W(C)$ aller Äquivalenzklassen ist ein Ring (Kompositionen von $\perp, \otimes$ induziert), und heißt der Wittring von $C$ (s. [4]). Das negative zu einer Äquivalenzklasse $(E)$ wird dabei durch den Raum $-E$ repräsentiert, der aus $E$ durch Änderung der Bilinearform $B$ zu $-B$ entsteht. Für eine natürliche Zahl $r>0$ schreiben wir für den Raum $r \times(-E)$ auch $(-r) \times E$. Für $r=0$ bezeichne $r \times E$ den Nullraum.

Theorem 2.1. Jedes Torsionselement der additiven Gruppe von $W(C)$ hat als Ordnung eine 2-Potenz.

Um dies zu beweisen, benötigen wir zunächst einen

Hilfssatz 2.2. $E$ und $M$ seien Räume über $C$. Die von den Normen von $E$ additiv erzeugte Normgruppe $\mathrm{g} E$ umfasse $\mathfrak{g} M ; M$ und $E \perp M$ seien metabolisch. Dann ist $k \times E$ metabolisch für jedes $k \geqq 3$.

Beweis. Es ist $E \perp M \cong E \perp r \times A(0,0)$ mit $r=\operatorname{dim} M$ (s. [4], 6.1.2). Wir können also $M=r \times A(0,0)$ voraussetzen. Sei $x_{1}, \ldots, x_{2 n}$ eine Basis von $E$ und $\alpha_{i}=n\left(x_{i}\right)$. Man überlegt sich leicht, daß für $k \geqq 2$ die metabolischen Räume $k \times E \perp k r \times A(0,0)$ und

$$
\stackrel{1}{i=1}^{2 n} A\left(\alpha_{i}, 0\right) \perp[k r+(k-2) n] \times A(0,0)
$$

dieselbe Normgruppe haben. Da in beiden Räumen mindestens eine hyperbolische Ebene enthalten ist, sind sie isomorph (s. Beweis von [4], 6.2.1). Für 
$k \geqq 3$ erhält man nun mit dem verallgemeinerten Kürzungssatz von O’Meara ([4], 6.1.3):

$$
k \times E \cong \underset{i=1}{2 n} A\left(\alpha_{i}, 0\right) \perp(k-2) n \times A(0,0) .
$$

Beweis von Theorem 2.1. Wir denken uns ein vorgegebenes Torsionselement von $W(C)$ durch einen eigentlichen Raum $E=\left(a_{1}, \ldots, a_{n}\right)$ repräsentiert. Zu jedem $n$-Tupel $\varepsilon=\left(\varepsilon_{1}, \ldots, \varepsilon_{n}\right)$ von Vorzeichen $\varepsilon_{i}= \pm 1$ bilden wir den Raum

$$
\pi(\varepsilon, a):=\bigotimes_{i=1}^{n}\left(1, \varepsilon_{i} a_{i}\right) \text {. }
$$

Ersichtlich ist $\left(a_{i}\right) \otimes \pi(\varepsilon, a) \cong\left(\varepsilon_{i}\right) \otimes \pi(\varepsilon, a)$ für $1 \leqq i \leqq n$. Daher gilt

$$
E \otimes \pi(\varepsilon, a) \sim \varphi(\varepsilon) \times \pi(\varepsilon, a)
$$

mit $\varphi(\varepsilon):=\varepsilon_{1}+\cdots+\varepsilon_{n}$.

Aus (2.3) erhalten wir durch Summation über alle $n$-Tupel $\varepsilon$

$$
2^{n} \times E \sim \frac{1}{\varepsilon} \varphi(\varepsilon) \times \pi(\varepsilon, a) .
$$

Es genügt zu zeigen, da $\beta$ jedes $\pi(\varepsilon, a)$ mit $\varphi(\varepsilon) \neq 0$ in $W(C)$ als Ordnung eine 2-Potenz hat. Nach (2.3) ist $\pi(\varepsilon, a)$ ein Torsionselement. Wir haben uns damit auf den Fall zurückgezogen, daß $E$ von der Form $\pi(\varepsilon, a)$ ist.

Sei also $E$ ein Pfisterraum und $m \times E \sim 0$ mit $m>0$. Wir können einen Raum

$$
M:=A\left(0, b_{1}\right) \perp \cdots \perp A\left(0, b_{r}\right)
$$

finden, so daß $m \times E \perp M$ metabolisch ist. Zu jedem $r$-Tupel $\eta=\left(\eta_{1}, \ldots \eta_{r}\right)$ von Vorzeichen bilden wir den Raum

Für jedes $\eta$ ist

$$
\pi(\eta, b)=\bigotimes_{i=1}^{r}\left(1, \pi_{i} b_{i}\right)
$$

$$
\mathfrak{g}[M \otimes \pi(\eta, b)]=\mathfrak{g} \pi(\eta, b) \subset \mathfrak{g}[E \otimes \pi(\eta, b)],
$$

also nach Hfs. 2.2 der Raum $3 m \times E \otimes \pi(\eta, b)$ metabolisch. Sei $2^{N}>3 m$. Nach Theorem $1.5 \mathrm{~d})$ ist $2^{N} \times E \otimes \pi(\eta, b)$ ein runder Raum. Er enthält einen Teilraum der Gestalt $(\lambda,-\lambda)$ mit $\lambda \in C^{*}$, besitzt also $(-1)$ als Ähnlichkeitsnorm. Für jedes $\eta$ ist somit $2^{N+1} \times E \otimes \pi(\eta, b)$ metabolisch. Summation über alle $\eta$ ergibt $2^{N+r+1} \times E \sim 0$.

q.e.d.

Wie bei Pfister ([11], S. 120) läßt sich auch hier das soeben bewiesene Theorem verschärfen zu

Satz 2.2. In $W(C)$ gibt es keine Nullteiler ungerader Dimension.

Satz 2.3. (vgl. [11], Satz 17, Satz 22). Das Nilradikal von $W(C)$ besteht aus den Torsionselementen gerader Dimension. 
Beweis (Witt). Wir setzen stillschweigend alle auftretenden Räume als eigentlich voraus.

a) Sei $\operatorname{dim} E=2 m, 2^{\mu} \times E \sim 0$. Wir zerlegen $E$ in Räume $E_{i} \cong\left(b_{i}\right) \otimes\left(1, a_{i}\right)$ $(i=1, \ldots m)$. Es ist

$$
E_{i}^{\otimes(\mu+1)} \cong 2^{\mu} \times\left(b_{i}^{\mu}\right) \otimes E_{i},
$$

also $E_{i}^{\otimes(\mu+1)} \otimes E \sim 0$. Daher $E^{\otimes(m \mu+2)} \sim 0$.

b) Sei $E^{\otimes r} \sim 0$ und $E \cong\left(a_{1}, \ldots, a_{n}\right)$ mit $a_{i} \in C^{*}$. Wir zeigen, daß $E$ ein Torsionselement von $W(C)$ liefert. Mit den im Beweis von Theorem 2.1 eingeführten Hilfsräumen $\pi(\varepsilon, a)$ gilt nach $(2.3)$

$$
\varphi(\varepsilon)^{r} \times \pi(\varepsilon, a) \sim E^{\otimes r} \otimes \pi(\varepsilon, a) \sim 0 .
$$

Die $\pi(\varepsilon, a)$ mit $\varphi(\varepsilon) \neq 0$ sind also Torsionselemente. Nach (2.4) ist auch $E$ in $W(C)$ Torsionselement.

\section{§ 3. Stufe eines lokalen Ringes}

In diesem Abschnitt sei $C$ ein lokaler Ring mit maximalem Ideal $\mathrm{m}$.

Satz 3.1. Es sei $2 \in C^{*}$ und $E$ ein isotroper bilinearer Pfister-Raum über $C$. Dann gibt es einen Pfister-Raum $P$ mit $E \cong(1,-1) \otimes P$.

Beweis. Sei $E=\left(1, a_{1}\right) \otimes \cdots \otimes\left(1, a_{n}\right)$ und ohne Einschränkung $n>1$. Wir benutzen im folgenden den Raum $V:=\left(\mathbb{Z}_{2}\right)^{n}$ über dem Körper $\mathbb{Z}_{2}$ mit 2 Elementen als Indexmenge. Sei $e_{1}, \ldots, e_{n}$ die Standardbasis von $V$. Zu jedem Vektor $x=e_{i_{1}}+\cdots+e_{i_{r}}\left(i_{1}<\cdots<i_{r}\right)$ bezeichne $a_{x}$ das Produkt $a_{i_{1}} \ldots a_{i_{r}}$. Nach Voraussetzung gibt es eine Gleichung

$$
\sum_{x \in V} a_{x} u_{x}^{2}=0
$$

mit Elementen $u_{x}$ von $C$, die nicht alle in $m$ liegen. Wir normieren die Gleichung so, da $\beta u_{0}=1$ ist. Für eine beliebige Hyperebene $W$ von $V$ bezeichne $E_{W}$ den in $E$ einbettbaren Pfister-Raum $\underset{x \in W}{\perp}\left(a_{x}\right)$ und $c_{W}$ das von $E_{W}$ dargestellte Element $\sum_{x \in W} a_{x} u_{x}^{2}$. Mit beliebig gewähltem $x \in V \backslash W$ und ebenfalls von $E_{W}$ dargestelltem $d_{W}$ läßt sich (3.2) schreiben als Gleichung

$$
c_{W}+a_{x} d_{W}=0 .
$$

Ist für mindestens eine Hyperebene $W$ das Element $c_{W}$ Einheit, so sind wir

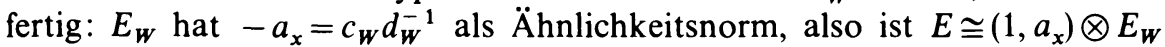
$\cong(1,-1) \otimes E_{W}$. Es können aber nicht alle $c_{W}$ in $m$ liegen. Denn dann erhielte man durch Summation über alle $W$ :

$$
\left(2^{n}-1\right) c_{0}+\left(2^{n-1}-1\right) \sum_{x \neq 0} c_{x} \in m
$$

mit $c_{x}:=a_{x} u_{x}^{2}$. Aus (3.2) würde $2^{n-1} c_{0} \in \mathrm{m}$ folgen, im Widerspruch zu $c_{0}=1$ und $2 \in C^{*}$. 
Aus diesem Satz 3.1 folgt sofort (Pfister, Witt)

Satz 3.3. Sei $2 \in C^{*}$ und $E$ ein Pfister-Raum.

(i) $E$ ist genau dann Torsionselement in $W(C)$, wenn ein Vielfaches $r \times E$ den Raum - E enthält.

(ii) Hat $E$ die Ordnung $2^{\mu+1}$ in $W(C)$ mit $\mu \geqq 0$, so ist $2^{\mu} \times E$ das kleinste Vielfache von $E$, das $-E$ enthält.

Beweis. Ist $-E$ in $2^{k} \times E$ enthalten, so ist $-1 \in N\left(2^{k} \times E\right)$, also $2^{k+1} \times E \sim 0$. Umgekehrt folgt aus $2^{\mu+1} \times E \sim 0$ wegen $2 \in C^{*}$, daß $2^{\mu} \times E \cong 2^{\mu} \times(-E)$ ist, und somit, daß $-E$ in $2^{\mu} \times E$ einbettbar ist. Damit ist (i) klar, sogar über einem semilokalen Ring $C$ mit $2 \in C^{*}$. Zum Beweis von (ii) ist nur noch auszuschließen, daß $-E$ in $\left(2^{\mu}-1\right) \times E$ enthalten ist. Wäre dies nun der Fall, so wäre $2^{\mu} \times E$ isotrop, aufgrund unseres Satzes 3.1 also schon $2^{\mu} \times E \sim 0$.

Als Stufe eines lokalen Ringes $C$ bezeichnen wir die kleinste Zahl $s$, zu der eine Relation

$$
-1=c_{1}^{2}+\cdots+c_{s}^{2}
$$

mit $c_{i} \in C$ existiert $(s=\infty$, falls -1 nicht Quadratsumme). Der Spezialfall $E=(1)$ von Satz 3.3 besagt, da $B$ im Falle $2 \in C^{*}$ der Ring $C$ genau dann endliche Stufe $s$ hat, wenn die Gruppe $W(C)$ endlichen Exponenten $h$ hat, und daß $s$ dann die 2-Potenz $\frac{h}{2}$ ist.

Ist $2 \in \mathrm{m}$, und $s$ endlich, $2^{k-1}<s \leqq 2^{k}$, so können wir immer noch folgern, daß $W(C)$ endlichen Exponenten $h$ hat, der $2^{k+1}$ teilt. Mir ist jedoch nicht klar, ob darüber hinaus ein Zusammenhang zwischen der Stufe und Eigenschaften von $W(C)$ besteht. Folgt aus einer Relation $2^{k} \times(1) \sim 0$, daß überhaupt ein Vielfaches $r \times(1)$ das Element -1 darstellt? Die Stufe braucht auch keine 2-Potenz zu sein, wie schon der Ring $\mathbb{Z} / 4 \mathbb{Z}$ der Stufe 3 zeigt.

Hilfssatz 3.4. Sei $2 \in \mathrm{m}$ und $r$ eine 2-Potenz. Dann folgt aus einer Relation $x_{1}^{2}+\cdots+x_{2 r}^{2}=0$ mit Elementen $x_{i} \in C$, die nicht alle in der gleichen Kongruenzklasse modm liegen, daß die Stufe $s \leqq r$ ist.

Beweis. Gibt es eine Teilsumme von $r$ Quadraten in $x_{1}^{2}+\cdots+x_{2 r}^{2}$, die eine Einheit $\varepsilon$ ist, so ergibt unsere Relation nach Division durch $\varepsilon$ eine Darstellung von -1 als Summe von $r$ Quadraten, denn nach 1.6 ist $G_{r}$ eine Gruppe. Liegen aber alle Teilsummen von $r$ Gliedern aus $x_{1}^{2}+\cdots+x_{2 r}^{2}$ in $m$, so müssen alle $x_{i}$ zueinander mod $m$ kongruent sein. Dieser Fall wurde ausgeschlossen. q.e.d.

Satz 3.5. Sei $2 \in \mathrm{m}$. $C$ habe endliche Stufe s. Dann hat $s$ die Gestalt $2^{k}$ oder $2^{k}-1$.

Beweis. Sei $2^{k-1}<s \leqq 2^{k}$. Wir haben eine Relation

$$
1+x_{2}^{2}+\cdots+x_{s+1}^{2}=0 \text {. }
$$

Ist $s \leqq 2^{k}-2$, so können wir Hilfssatz 3.4 anwenden mit $x_{s+2}=\cdots=x_{2 r}=0$. Wir erhalten den Widerspruch $s \leqq 2^{k-1}$.

Beispiel 3.6. Ausgehend von dem Ring $C=\mathbb{Z} / 4 \mathbb{Z}$ lassen sich leicht lokale Ringe der Stufe 3 mit großen Restklassenkörpern konstruieren. Wir betrachten 
allgemeiner einen lokalen Ring $(C, m)$ mit $2 \lambda=\lambda^{2}=0$ für alle $\lambda \in m$. Sei $D$ die Lokalisierung $C\left[t_{1}, \ldots, t_{n}\right]_{\mathfrak{p}}$ des Polynomringes in $n$ Variablen $t_{i}$ über $C$ nach einem Primideal $\mathfrak{p}$, das $\mathfrak{m} C\left[t_{1}, \ldots t_{n}\right]$ umfaßt. Dann ist $D$ eine Erweiterung von $C$, welche dieselbe Stufe wie $C$ besitzt. Sei nämlich

$$
-1=\left(g^{-1} f_{1}\right)^{2}+\cdots+\left(g^{-1} f_{s}\right)^{2}
$$

eine Gleichung in $D$ mit $f_{i}, g \in C\left[t_{1}, \ldots t_{n}\right], g \notin \mathfrak{p}$. Mit einem weiteren nicht in $\mathfrak{p}$ gelegenen Polynom $h$ haben wir eine Gleichung

$$
-(g h)^{2}=f_{1}^{2}+\cdots+f_{s}^{2} .
$$

Mit $\tilde{g}$ bzw. $\tilde{f}_{i}$ bezeichnen wir die Polynome, die aus $g h$ und den $f_{i} h$ entstehen, indem wir alle Glieder mit Koeffizienten in $m$ einfach weglassen. Wegen unserer Voraussetzung über $m$ ist $\tilde{g}^{2}=(g h)^{2}, \tilde{f}_{i}^{2}=\left(f_{i} h\right)^{2}$ und sicher $\tilde{g} \neq 0$, da $\operatorname{sogar} \tilde{g} \notin \mathfrak{p}$. Wir vergleichen nun in der Gleichung

$$
-\tilde{g}^{2}=\tilde{f}_{1}^{2}+\cdots+\tilde{f}_{s}^{2}
$$

die Koeffizienten des höchsten Monomes bzgl. der lexikographischen Anordnung, das in einem der $\tilde{f}_{i}^{2}$ wirklich auftritt, und sehen, da $B-1$ schon in $C$ Summe von $s$ oder weniger Quadraten ist.

\section{§ 4. Annullatoren runder Formen}

Wir nennen zwei quadratische Räume $E, F$ äquivalent, und schreiben $E \sim F$, falls es hyperbolische quadratische Räume $M, N$ gibt mit $E \perp M \cong F \perp N$. Die Äquivalenzklassen aller quadratischen Räume über $C$ bilden eine Gruppe, die Wittgruppe $W q(C)$ (vgl. [1], Chapter V). $W q(C)$ ist durch das Tensorprodukt ein Modul über dem Wittring $W(C)$ der bilinearen Räume. Jeder quadratische Raum $E$ ist orthogonale Summe $N \perp E_{0}$ eines hyperbolischen Raumes $N$ mit einem anisotropen Raum $E_{0}(\mathrm{vgl}$. [4], 3.2.1). Da nun für quadratische Räume über semilokalem $C$ die Kürzungsregel gilt ([5], s. auch [12]), ist - wie im Körperfalle - die Äquivalenzklasse von $E$ durch die Isomorphieklasse von $E_{0}$ gekennzeichnet. Wir nennen $E_{0}$ einen Kernraum von $E$.

Theorem 4.1. Sei $E$ runder quadratischer Raum über $C$ und $E$ nicht hyperbolisch. Es gelte eine der folgenden Voraussetzungen:

a) $\operatorname{dim} E>2,\left|C / m_{i}\right|>2$ für alle $m_{i}$;

b) $\operatorname{dim} E=2,\left|C / \mathrm{m}_{i}\right|>3$ für alle $\mathrm{m}_{i}$, für jedes $\mathrm{m}_{i}$ mit $\left|C / \mathrm{m}_{i}\right|=4$ ist $E / \mathrm{m}_{i} E$ anisotrop.

Behauptung. Das Annullatorideal von $(E) \in W q(C)$ in $W(C)$ wird durch die Räume $(1,-\lambda)$ mit $\lambda \in N(E)$ erzeugt.

Beweis. Wir können uns auf den Fall zurückziehen, daß $E$ anisotrop ist. Ist $E$ nämlich isotrop und rund und $E_{0}$ ein Kernraum von $E$, so folgt aus $N(E)=q\left(E^{*}\right)=C^{*}$ mit der Kürzungsregel $N\left(E_{0}\right)=C^{*}$, also a fortiori, daß $E_{0}$ rund ist. 
Sei ab jetzt $E$ anisotrop und $I$ das von den $(1,-\lambda)$ mit $\lambda \in N(E)$ in $W(C)$ erzeugte Ideal, das sicherlich im Annullatorideal Ann $E$ enthalten ist. Für $b \in C^{*}$ und eine Einheit $c=\xi^{2} q(x)+b \eta^{2} q(y)$ mit $x, y \in E^{*} ; \xi, \eta \in C$ gilt:

$$
(1, b) \equiv(c) \otimes(1, b) \bmod I .
$$

In der Tat wird $E$ von $(1,-c) \otimes(1, b)$ annulliert (s. Hilfssatz 1.3) und damit auch von dem dazu mod $I$ kongruenten Raum

$$
F=(q(x), b q(y),-c,-b c) .
$$

Da der Teilraum $(q(x), b q(y),-c)$ isotrop ist, hat $F$ die Gestalt $N \perp G$ mit metabolischem $N$ und binärem eigentlichen $G$, und es ist $F \equiv G \equiv 0 \bmod I$. Kongruenzen der Art (4.2) werden wir im folgenden ohne besondere Erwähnung häufig ausnutzen.

Angenommen, Ann $E \neq I$. Sei $F=\left(b_{1}, \ldots, b_{n}\right)$ ein eigentlicher Raum minimaler Dimension $n$ mit $F \neq 0$ mod $I, F \otimes E \sim 0$. Der Raum $\left(b_{2}, \ldots, b_{n}\right) \otimes E \operatorname{mu} B$ einen zu $\left(-b_{1}\right) \otimes E$ isomorphen Kernraum besitzen. Daher gibt es eine Gleichung

$$
b_{1}+b_{2} q\left(x_{2}\right)+\cdots+b_{n} q\left(x_{n}\right)=0
$$

mit $x_{i} \in E$. Wir wollen zunächst $F$ modulo $I$ zu einem Raum $F^{\prime}=\left(b_{1}^{\prime}, \ldots, b_{n}^{\prime}\right)$ abändern, so daß sogar

$$
b_{1}^{\prime}+b_{2}^{\prime}+\cdots+b_{n}^{\prime}=0
$$

ist. Aufgrund von Korollar 1.4 gibt es ein Produkt $c$ von Einheiten der Gestalt $\xi^{2} q(x)+b_{1}^{-1} b_{2} \eta^{2} q(y)$ mit $x, y \in E^{*}, \xi, \eta \in C$, so da $\beta c\left(b_{1}+b_{2} q\left(x_{2}\right)\right)$ sich in der Gestalt $b_{1} q\left(x_{1}^{\prime}\right)+b_{2} q\left(x_{2}^{\prime}\right)$ mit $x_{1}^{\prime}, x_{2}^{\prime} \in E^{*}$ schreiben läßt. Mit $b_{i}^{\prime}=c^{-1} b_{i} q\left(x_{i}^{\prime}\right)$ $(i=1,2)$ gilt nach der anfangs gemachten Bemerkung

$$
\left(b_{1}, \ldots, b_{n}\right) \equiv\left(c^{-1} b_{1}, c^{-1} b_{2}, b_{3}, \ldots b_{n}\right) \equiv\left(b_{1}^{\prime}, b_{2}^{\prime}, b_{3}, \ldots b_{n}\right)
$$

$\bmod I$ und es ist $b_{1}^{\prime}+b_{2}^{\prime}+b_{3} q\left(x_{3}\right)+\cdots+b_{b} q\left(x_{n}\right)=0$. Man setze das Verfahren fort.

Wir können also annehmen, daß für unseren Raum $F=\left(b_{1}, \ldots, b_{n}\right)$ die Summe $b_{1}+\cdots+b_{n}=0$ ist. Sei $e_{1}, \ldots, e_{n}$ eine Orthogonalbasis von $F$ mit $n\left(e_{i}\right)=b_{i}$. Wir wollen den isotropen Vektor $u=e_{1}+\cdots+e_{n}$ in eine metabolische Ebene $N \subset F$ einbetten, so daß das orthogonale Komplement $N^{\perp}=G$ eigentlich ist. Es ist dann $F=N \perp G$ und wir erhalten den gewünschten Widerspruch $G \otimes E \sim 0$ zur Minimalität von $\operatorname{dim} F$. Dieses Problem ist lösbar, wenn es für jedes maximale Ideal $\mathrm{m}$ von $C$ gelingt, das Bild $\bar{u} \in F / \mathrm{m} F$ in eine metabolische Ebene von $F / \mathrm{m} F$ mit eigentlichem orthogonalen Komplement einzubetten, und das wiederum ist genau dann möglich, wenn es einen zu $\bar{u}$ orthogonalen anisotropen Vektor in $F / \mathrm{m} F$ gibt.

Angenommen, für ein maximales Ideal $m$ seien alle zu $\bar{u}$ orthogonalen Vektoren isotrop. Insbesondere sind die Bilder der Vektoren $b_{j} x_{i}-b_{i} x_{j}$ für $i \neq j$ alle isotrop, d. h. $b_{i} \equiv-b_{j} \bmod \mathrm{m}$. Da sicherlich $n \geqq 3$ ist, muß $2 \in \mathrm{m}$ und 
$b_{1} \equiv \cdots \equiv b_{n} \bmod m$ sein. In diesem Falle ist $B(u, x)^{2} \equiv n(x) b_{1}$ für alle $x \in E$ und unsere Aufgabe tatsächlich unlösbar.

Wir müssen also den Raum $F=\left(b_{1}, \ldots, b_{n}\right)$ zuvor $\bmod I$ so abändern, daß immer noch $\sum_{i}^{n} b_{i}=0$ ist, aber für kein $m$ mit $2 \in m$ alle $b_{i}$ zueinander kongruent sind. Dazu gehen wir zunächst zu $\left(b_{1}^{-1}\right) \otimes F$ über, nehmen also ohne Einschränkung der Allgemeinheit $b_{1}=1$ an. Sei $c$ eine Einheit der Gestalt $\xi^{2} q(x)+\eta^{2} b_{2} q(y)$ mit $x, y \in E^{*}, \xi, \eta \in C$. Dann ist

$$
c\left(1+b_{2}\right)=q\left(x^{\prime}\right)+b_{2} q\left(y^{\prime}\right)
$$

mit $x^{\prime}=\xi x-\eta b_{2} y, y^{\prime}=\xi x+\eta y$. Angenommen, $q\left(x^{\prime}\right)$ und $q\left(y^{\prime}\right)$ sind Einheiten. Dann gilt mit $b_{1}^{\prime}=c^{-1} q\left(x^{\prime}\right), b_{2}^{\prime}=c^{-1} b_{2} q\left(y^{\prime}\right)$ wie oben:

$$
F \equiv\left(c^{-1}, c^{-1} b_{2}, b_{3}, \ldots, b_{n}\right) \equiv\left(b_{1}^{\prime}, b_{2}^{\prime}, b_{3}, \ldots, b_{n}\right)
$$

$\bmod I$ und es ist $n \geqq 3$ und auch

$$
b_{1}^{\prime}+b_{2}^{\prime}+b_{3}+\cdots+b_{n}=0 .
$$

Wir wollen nun $x, y \in E^{*}$ und $\xi, \eta \in C$ so wählen, daß neben den für alle maximalen Ideale $\mathrm{m}$ zu erfüllenden Bedingungen $q\left(x^{\prime}\right) \neq 0, q\left(y^{\prime}\right) \neq \equiv, c$ 丰 0 , an den $\mathrm{m}$ mit $2 \in \mathrm{m}$ zusätzlich nicht $b_{1}^{\prime} \equiv b_{2}^{\prime} \equiv b_{3}$ ist. Dann sind wir fertig. Ist $2 \notin \mathrm{m}$ oder nicht $1 \equiv b_{2} \equiv b_{3}$, so kommen wir mit $\xi \equiv 1$, irgendwelchen anisotrop vorgeschriebenen Bildern für $x, y$ in $E / \mathrm{m} E$ und $\eta \equiv 0$ zum Ziel. Sei jetzt $m$ ein maximales Ideal mit $1 \equiv b_{2} \equiv b_{3}(\mathfrak{m})$ und $2 \in \mathfrak{m}$. Der Raum $E=E / \mathfrak{m} E$ enthält eine Ebene $\cong A[1, \bar{a}]$ mit zunächst beliebigem $\bar{a} \in C / \mathrm{m}$, das wir aber durch Abänderung des entsprechenden Basiselementes der Ebene immer zu $\neq 0$ machen können, weil $|C / \mathrm{m}|>2$ ist. Wir schreiben die Bilder von $x, y$ in $E / \mathrm{m} E$ als Vektoren mit der Wertetabelle $A[1, \bar{a}]$ vor und weiter $\xi \equiv 1$. Dann haben wir für das Bild $\bar{\eta}$ von $\eta$ in $C / m$ folgende Vorschriften:

$$
1+\bar{a} \bar{\eta}^{2} \neq 0, \quad 1+\bar{\eta}+\bar{a} \bar{\eta}^{2} \neq 0, \quad \bar{\eta} \neq 0,
$$

wobei die Forderung $\bar{\eta} \neq 0$ gleichwertig zu $b_{1}^{\prime} \neq 1$ ist. Diese Vorschriften lassen sich für $|C / m|>4$, also $\geqq 8$, ersichtlich erfüllen und für $|C / m|=4$ genau dann, wenn $\bar{a} \neq 1$ ist. Sei jetzt $|C / \mathrm{m}|=4$. Ist $\operatorname{dim} E>2$, so ist $E \cong A[1, \bar{a}] \perp G$ mit universellem $G$, und wir können diese Zerlegung leicht so abändern, da $\bar{a} \neq 1$ ist. Ist $\operatorname{dim} E=2$ und $\bar{a}=1$, so ist $\bar{E} \cong A[0,0]$ und man sieht leicht, daß der hier eingeschlagene Weg keinen Erfolg hat.

q.e.d.

Bemerkung 4.4. Ist $C$ ein Körper, so sind die über $C$ in Theorem 4.1 gemachten Einschränkungen überflüssig. Es ist dann nämlich a priori klar, da $\beta$ der bilineare Raum $F$ im Beweis anisotrop sein muß, auch für Charakteristik 2. In der Gleichung (4.3) kann man die Glieder mit $q\left(x_{i}\right)$ Nichteinheit, also $=0$, weglassen und sofort den Widerspruch herbeiführen [Witt, unveröffentlicht]. Über einem Körper $C$ der Charakteristik 2 wird auch der Annullator eines runden Bilinearraumes $E$ durch eigentliche binäre Räume erzeugt. Dies ergibt sich ähnlich dem soeben skizzierten Beweis, weil jetzt auch in jeder Ähnlichkeitsklasse von Bilinearräumen genau eine Isomorphitklasse von anisotropen 
Räumen liegt ([4], 8.2.1, [15]). \{Über beliebigen lokalen Ringen mit 2 Nichteinheit braucht das nicht der Fall zu sein, s. z. B. [4], 9.3.8.

Aus Theorem 4.1 folgt mit Satz 1.2, daß ein Torsionselement der Wittgruppe $W q(C)$, das durch einen runden Raum repräsentiert werden kann, 2-Potenz-Ordnung hat, sofern alle $\left|C / \mathrm{m}_{i}\right|>2$ sind. Meines Wissens ist im Falle $2 \notin C^{*}$ nicht bekannt, ob jedes Torsionselement von $W q(C)$ 2-Potenz-Ordnung hat.

\section{Literatur}

1. Bass, H.: Lectures on topics in algebraic $K$-theory. Tata Inst. Fund. Res., Bombay 1967.

2. Bourbaki, N.: Algèbre, Chap. 9, Formes sesquilinéaires et formes quadratiques. Paris: Hermann 1959.

3. Knebusch, M., Rosenberg, A., Ware, R.: Structure of Wittrings, quotients of abelian group rings, and orderings of fields. Bull. Amer. Math. Soc. 77, 205-210 (1971).

4. - Grothendieck- und Wittringe von nicht ausgearteten symmetrischen Bilinearformen. Sitzber. Heidelberg. Akad. Wiss. 1969/70, 3 Abb., 93-157 (als Einzelheft im Handel, BerlinHeidelberg-New York: Springer 1969/70).

5. - Isometrien über semilokalen Ringen. Math. Z. 108, 255-268 (1969).

6. - Bemerkungen zur Theorie der quadratischen Formen über semilokalen Ringen. Math. Inst. d. Universität des Saarlandes, 1969 (auf Wunsch beim Autor erhältlich).

7. Lorenz, F.: Quadratische Formen über Körpern. Lecture notes in math., 130. Berlin-Heidelberg-New York: Springer 1970.

8. O'Meara, O.T.: Introduction to quadratic forms. Berlin-Göttingen-Heidelberg: Springer 1963.

9. Pfister, A.: Zur Darstellung von -1 als Summe von Quadraten in einem Körper. J. London M. S. 40, 159-165 (1965).

10. - Multiplikative quadratische Formen. Arch. Math. 16, 363-370 (1965).

11. - Quadratische Formen in beliebigen Körpern. Invent. math. 1, 116-132 (1966).

12. Roy, A.: Cancellation of quadratic forms over commutative rings. J. Algebra 10, 286-298 (1968).

13. Scharlau, W.: Zur Pfisterschen Theorie der quadratischen Formen. Invent. math. 6, 327-328 (1969).

14. - Quadratic forms. Queen's papers on pure appl. math. 22. Queen's University, Kingston, Ontario 1969.

15. Milnor,J.: Symmetric inner product spaces in characteristic 2, preprint Inst. Adv. Study. Princeton 1970.

\author{
Dr. Manfred Knebusch \\ Mathematisches Institut \\ der Universität des Saarlandes \\ BRD-6600 Saarbrücken 15, Bau 27 \\ Deutschland
}

